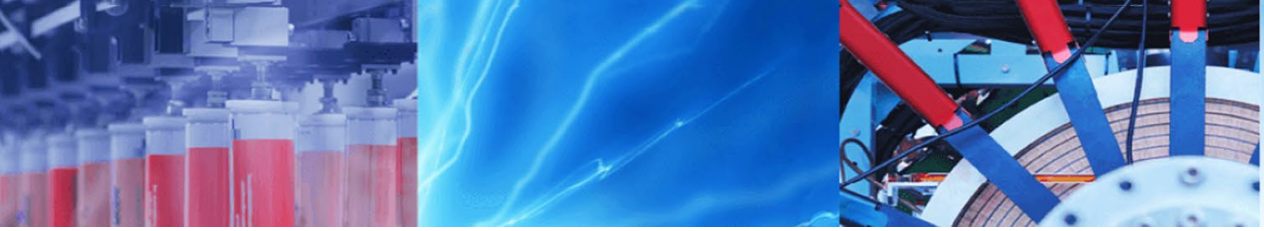

Research Article

\title{
Variations in magnetic properties caused by size dispersion and particle aggregation on $\mathrm{CoFe}_{2} \mathrm{O}_{4}$
}

\author{
Fernando Arteaga-Cardona ${ }^{1}$ (D) Nery Gabriela Martha-Aguilar ${ }^{1} \cdot$ José Octavio Estevez $^{2} \cdot$ Umapada Pal $^{1}$. \\ Miguel Ángel Méndez-Rojas ${ }^{3}$. Ulises Salazar-Kuri ${ }^{1}$
}

(c) Springer Nature Switzerland AG 2019

\begin{abstract}
Size dispersion and particle aggregation are the key parameters that affect the magnetic properties at nanoscale due to interparticle interactions. However, few efforts have been devoted so far to understand how these parameters affect the magnetic properties of nanoparticles. Here, we experimentally demonstrate how the magnetic properties such as magnetic saturation $\left(M_{\mathrm{s}}\right)$, coercivity $\left(H_{\mathrm{c}}\right)$, Curie temperature $\left(T_{\mathrm{c}}\right)$, and blocking temperature $\left(T_{\mathrm{B}}\right)$ of cobalt ferrite $\left(\mathrm{CoFe}_{2} \mathrm{O}_{4}\right)$ nanoparticles having the same composition and near-average size are affected by size dispersion $(\sigma)$ and aggregation. Cobalt ferrite nanoparticles of similar average sizes but different size dispersions and aggregations were fabricated through different synthesis routes. The results clearly demonstrate that just by reducing the size dispersion and aggregation, it is possible to modify the magnetic properties, e.g., achieving a superparamagnetic state of cobalt ferrite even under applied magnetic field as low as 100 Oe as indicated by ZFC measurements. The Stoner-Wohlfarth model with thermal agitation was used to simulate the blocking temperature of the different size dispersion and aggregation nanoparticles confirming that low size dispersion and nonaggregated particles have great influence to achieve the superparamagnetic state, especially for high coercivity materials such as cobalt ferrite.
\end{abstract}

Keywords Superparamagnetism · Curie temperature · Saturation magnetization · Blocking temperature

\section{Introduction}

The control of the materials properties at the nanoscale is an enormous challenge for the materials scientists [ 1 , $13,40]$, as the specific application of nanomaterials needs their highly controlled fabrication [41]. Chemical synthetic routes have attracted much attention for the synthesis of nanoparticles, as they are cheaper alternatives than other physical synthetic routes $[2,27,35,39]$. However, most of the time the material presents poor crystallinity, high defect content, a considerable size dispersion $(\sigma)$, and particle aggregation. All these factors are important to consider as they affect strongly their magnetic behavior. One example can be that the interparticle interactions in agglomerated or assembled magnetic nanoparticles strongly affect their magnetic and other physical properties, mainly by increasing the overall effective anisotropy $\left(K_{\text {eff }}\right)[22]$.

Superparamagnetism is due to the small size of the nanoparticles; each one acts as one individual crystalline magnetic domain with a net spin. The net magnetization of the individual nanoparticle is a consequence of the addition of the magnetic moments of hundreds of atoms, and thus, the result is thousands of times greater than a normal

Fernando Arteaga-Cardona, fernando.artc@gmail.com; $₫$ Ulises Salazar-Kuri, usalazar@ifuap.buap.mx| ${ }^{1}$ Instituto de Física, Benemérita Universidad Autónoma de Puebla, Apdo. Postal J-48, 72570 Puebla, Pue, Mexico. ${ }^{2}$ Departamento de Materia Condensada/Instituto de Física UNAM, Circuito de la Investigación Científica Ciudad Universitaria, C.P. 04510 Ciudad de Mexico, Mexico. ${ }^{3}$ Departamento de Ciencias Químico-Biológicas, Universidad de las Américas Puebla, ExHda. Sta. Catarina Mártir s/n, San Andrés Cholula, Puebla 72810, Mexico.

SN Applied Sciences (2019) 1:412 | https://doi.org/10.1007/s42452-019-0447-y 
paramagnetic material [33]. Depending on its nature, this state cannot be achieved for nanoparticles with sizes above $20 \mathrm{~nm}[29,42]$.

Being in the superparamagnetic state, nanoparticles are able to flip their magnetic spin according to the external applied magnetic field following the Néel relaxation model $\left(\tau=\tau_{0} \exp \left(E_{\mathrm{B}} / E_{\mathrm{T}}\right)\right.$, being $\tau_{0}$ the attempt time) over time, as long as the energy barrier $\left(E_{\mathrm{B}}=K_{\text {eff }} V \sin ^{2}(\theta), V\right.$ being the volume of the nanoparticles, and $\theta$ the angle between the magnetization and the easy axis) is lower than the thermal energy $\left(E_{T}=k_{B} T\right)$. As a result, superparamagnetic nanoparticles are excellent candidates for several applications, such as sensors and medical applications $[14,16$, $26,43]$.

Nevertheless, particle aggregation and size dispersion are recurrent problems for all nanomaterials; however, disaggregation of non-magnetic nanomaterials can be easily achieved, but not for magnetic materials. Aggregation can drastically affect the magnetic properties as it changes the effective anisotropy of the sample due to changes in the dipole energy by the extreme contact ( $E_{\mathrm{d}} \approx \mu_{0} \mu^{2} / 4 \pi d^{3}$, being $\mu_{0}$ the permeability of free space, and $\mu$ the average magnetic moment and $d$, interparticle distance) $[4,9]$.

The dispersion and aggregation of magnetic nanoparticles generate uncertainty to the measured magnetic properties as there are numerous nanoparticles with different sizes, shapes, and interactions among themselves, resulting in a non-homogeneous $E_{\mathrm{B}}$, which cannot be used as the overall $E_{\mathrm{B}}$ for all the samples $[10,36]$.

One of the most used chemical routes for the synthesis of magnetic nanomaterials is co-precipitation [10, 28]. However, in most of the cases, this route produces nanoparticles with a broad range size dispersion and heavy aggregation [17]. Most papers usually just report a histogram with a standard deviation $(\sigma)$ to show how much the sample deviates from monodispersion, as truly monodisperse samples are rare and considerably difficult to achieve [24]. In addition, the aggregation influence on the magnetic properties measured for the overall material is rarely considered to explain the magnetic results $[4,27,33]$.

Here, cobalt ferrite nanoparticles of near-average size, but with different particle aggregation and size dispersion degrees, were synthesized by using different synthetic routes. Cobalt ferrite was chosen for the present study because it is a popular magnetic material with high anisotropy. Due to this anisotropy, small variations in size result in drastic changes in the magnetic properties [23], especially in the blocking temperature $\left(T_{\mathrm{B}}\right)$, as $T_{\mathrm{B}}$ for the cobalt ferrite is rarely seen.

\section{Materials and methods}

Three different synthetic routes were followed to obtain nanoparticles with distinct aggregations and size dispersions: co-precipitation of metal nitrates followed by a thermal treatment; co-precipitation of metal chlorides followed by an acid treatment; and thermal decomposition of metal acetylacetonates in high-boiling-point organic solvent. These preparation routes were chosen as it has been reported that metal nitrates yield higher size dispersion and aggregation than their corresponding chlorides [19], while co-precipitation using metal chlorides is a widely used synthetic method for metal oxide particles and produces materials with both large size dispersion and aggregation; finally, the thermal decomposition of metal acetylacetonates is a well-known route that allows the preparation of small-sized nanoparticle with both low dispersion degree and aggregation.

\subsection{Co-precipitation of nitrates followed by a thermal treatment $\left(\mathrm{CoFe}_{2} \mathrm{O}_{4} \mathrm{TT}\right)$}

Stoichiometric amounts of $\mathrm{Co}\left(\mathrm{NO}_{3}\right)_{2} \cdot 6 \mathrm{H}_{2} \mathrm{O}(5 \mathrm{mmol})$ and $\mathrm{Fe}\left(\mathrm{NO}_{3}\right)_{3} \cdot 9 \mathrm{H}_{2} \mathrm{O}(10 \mathrm{mmol})$ were dissolved in deionized water and heated up to $70^{\circ} \mathrm{C}$, and then, a $2 \mathrm{M}$ solution of $\mathrm{NaOH}$ was slowly added to the metal ion solution. The solution was left stirring for $1 \mathrm{~h}$ after $\mathrm{NaOH}$ was added. The final measured $\mathrm{pH}$ was 14 . After this time, the supernatant was decanted and the precipitate was washed several times with distilled water. The obtained sample was heated at $80^{\circ} \mathrm{C}$ for several hours, and then the temperature was increased up to $500^{\circ} \mathrm{C}$ for $1 \mathrm{~h}$ [36].

\subsection{Co-precipitation of chlorides followed by an acid treatment $\left(\mathrm{CoFe}_{2} \mathrm{O}_{4}\right.$ acid)}

Stoichiometric amounts of $\mathrm{CoCl}_{2} \cdot 6 \mathrm{H}_{2} \mathrm{O}(5.0 \mathrm{mmol})$ and $\mathrm{FeCl}_{3} .6 \mathrm{H}_{2} \mathrm{O}(10.0 \mathrm{mmol})$ were dissolved in $5 \mathrm{~mL}$ deionized water with $250 \mu \mathrm{L}$ of $\mathrm{HCl}(37 \%)$ and heated up to $70{ }^{\circ} \mathrm{C}$. Then a second solution of $2 \mathrm{M}$ solution of $\mathrm{NaOH}$ was heated to $100{ }^{\circ} \mathrm{C}$. The solution with the metallic cations was rapidly added to the hot $\mathrm{NaOH}$ solution. The mixture was left stirring for $30 \mathrm{~min}$, and the final PH was 14 . After this time, the supernatant was decanted and the obtained precipitate was washed several times with distilled water. Without completely drying, $15 \mathrm{~mL}$ of a $2 \mathrm{M}$ solution of $\mathrm{HNO}_{3}$ was added to the black precipitate and left stirring at room temperature for $15 \mathrm{~min}$; after which the acid was decanted. Twenty milliliters of a $1 \mathrm{M}$ solution of $\mathrm{Fe}\left(\mathrm{NO}_{3}\right)_{3} \cdot 9 \mathrm{H}_{2} \mathrm{O}$ was then added and left stirring at boiling temperature for $30 \mathrm{~min}$. After this, the sample was 
left cooling at room temperature and the supernatant was decanted [6]. Finally, another $15 \mathrm{~mL}$ of a $2 \mathrm{M}$ solution of $\mathrm{HNO}_{3}$ was added to the precipitate and left stirring for $15 \mathrm{~min}$. After the $15 \mathrm{~min}$, the acid was decanted and the black precipitate was washed with acetone and some water was added to form a stable ferrofluid [3].

\subsection{Thermal decomposition of metal acetylacetonates ( $\mathrm{CoFe}_{2} \mathrm{O}_{4}$ TD)}

In a round-bottomed flask, $0.2 \mathrm{~g}(0.55 \mathrm{mmol})$ of $\mathrm{Co}(\mathrm{acac})_{3}$, $0.396 \mathrm{~g}(1.1 \mathrm{mmol})$ of $\mathrm{Fe}(\mathrm{acac})_{3}, 200 \mu \mathrm{L}$ of ethyleneglycol, $2 \mathrm{~mL}$ of oleic acid, and $0.476 \mathrm{~g}$ of CTAB were added to $20 \mathrm{~mL}$ of 1 -octadecene. The mixture was left under stirring at $100^{\circ} \mathrm{C}$ for $15 \mathrm{~min}$ to get rid of all the possible water in the flask. After $15 \mathrm{~min}$, the mixture was heated at $190^{\circ} \mathrm{C}$ for $1 \mathrm{~h}$. The color of the mixture slowly changed from reddish to deep black. After the reaction time finished, the mixture was left cooling at room temperature. The formed precipitate was collected and washed several times with ethanol. Finally, the collected sample was re-dispersed in isooctane for storage to avoid the aggregation of the nanoparticles [27].

\section{Characterization}

Size, morphology, and size dispersion of the obtained nanostructures were analyzed by TEM using a Jeol JEM2010F high-resolution transmission electron microscope (HRTEM). The samples for TEM were prepared by dispersing the samples $\mathrm{CoFe}_{2} \mathrm{O}_{4} \mathrm{TT}$ and $\mathrm{CoFe}_{2} \mathrm{O}_{4}$ acid in water, and $\mathrm{CoFe}_{2} \mathrm{O}_{4}$ TD sample in hexane, placing one droplet of each over a carbon-coated copper grid, followed by drying at room temperature. The size distribution histograms were obtained by measuring the size of over 300 nanoparticles for each sample and fitting the histogram to a lognormal distribution [20]. EDS of the samples was carried out in an ultra-high-resolution scanning electron microscope MAIA with field emission gun at $15 \mathrm{keV}$.

Hydrodynamic volume and size dispersion of the samples $\mathrm{CoFe}_{2} \mathrm{O}_{4}$ acid and $\mathrm{CoFe}_{2} \mathrm{O}_{4}$ TD were obtained by using a Nanotrac Wave II from Microtrac Inc. The sample $\mathrm{CoFe}_{2} \mathrm{O}_{4}$ acid was measured by diluting the sample in distilled water (refractive index, 1.33); meanwhile, the sample $\mathrm{CoFe}_{2} \mathrm{O}_{4}$ TD was diluted in toluene (refractive index, 1.50). The refraction index used for both $\mathrm{CoFe}_{2} \mathrm{O}_{4}$ samples was 2.42. DLS measurement was not performed on $\mathrm{CoFe}_{2} \mathrm{O}_{4}$ TT sample, because the nanoparticles in the sample were so aggregated that they precipitate almost immediately from their colloidal dispersion, thus obtaining non-reliable and non-reproducible results.
The identification of the crystals phases of the fabricated cobalt ferrite nanostructures was performed by recording their X-ray diffraction patterns in a PANalytical Empyrean diffractometer using copper $K a$ radiation at $45 \mathrm{kV}$ and $40 \mathrm{~mA}$; the data were collected from $20^{\circ}$ to $80^{\circ}$ in $2 \theta$. The average crystallite size was determined by using the Scherrer equation $[21,30]$ :

$D=\frac{\alpha \lambda}{\beta \cos \theta}$

where $a$ is a shape constant with a typical value of 0.9 for quasi-spherical nanoparticles, $\lambda$ is the measure wavelength (1.5406 $\AA$ ), $\beta$ represents the FWHM of the (311) peak and $\theta$ is the half of the angle where the (311) peak appears.

Thermogravimetric analysis was used to determine the organic mass from sample $\mathrm{CoFe}_{2} \mathrm{O}_{4}$ TD and the ferromagnetic Curie temperature for all the samples using a TA instruments SDT 650 simultaneous thermal analyzer under a nitrogen flux of $5 \mathrm{~mL} / \mathrm{min}$, from room temperature up to $800^{\circ} \mathrm{C}$. A permanent magnet ( $B=50 \mathrm{Oe}$ ) was placed on top to reduce the weight of the sample by $2-5 \%$, recording both heating and cooling data [18]. To assure the adsorbed water or other organic components at the surface of the nanoparticles do not contribute to the recorded signals producing errors in the estimated Curie temperature of the samples, the measurements were repeated for the TG and DSC immediately after finishing the first ones, keeping all parameters the same.

Magnetometry measurements were carried out in a vibrating sample magnetometer VSM attached to a Dynacool 9 physical properties measurement system (PPMS) from Quantum design. Magnetization hysteresis curves were recorded under an external magnetic field up to $2 \mathrm{~T}$ at 10, 100, 300, and $350 \mathrm{~K}$. The zero-field-cooled (ZFC) and field-cooled $(F C)$ magnetization curves were measured from $10 \mathrm{~K}$ to $350 \mathrm{~K}$ with an external magnetic field of 100 Oe. The blocking temperature for the sample measured at 100 Oe was obtained by the maximum value of the ZFC curve.

\section{Results and discussion}

\subsection{Size, morphology and dispersion}

According to the selected preparation routes, $\mathrm{CoFe}_{2} \mathrm{O}_{4}$ magnetic nanoparticles with near-average size but three different dispersion and aggregation degrees were obtained as it is seen from the TEM images (Fig. 1). The largest aggregation and size dispersion observed were for the sample prepared by co-precipitation followed by a heat treatment $\left(\mathrm{CoFe}_{2} \mathrm{O}_{4} \mathrm{TT}\right)$. Nanoparticles sizes ranged from 6 to $34 \mathrm{~nm}(\sigma=0.93)$. Also, as these are ferrimagnetic 
Fig. 1 TEM images of the samples a $\mathrm{CoFe}_{2} \mathrm{O}_{4} \mathrm{TT}$, $\mathbf{b} \mathrm{CoFe}_{2} \mathrm{O}_{4}$ acid, c $\mathrm{CoFe}_{2} \mathrm{O}_{4}$ TD

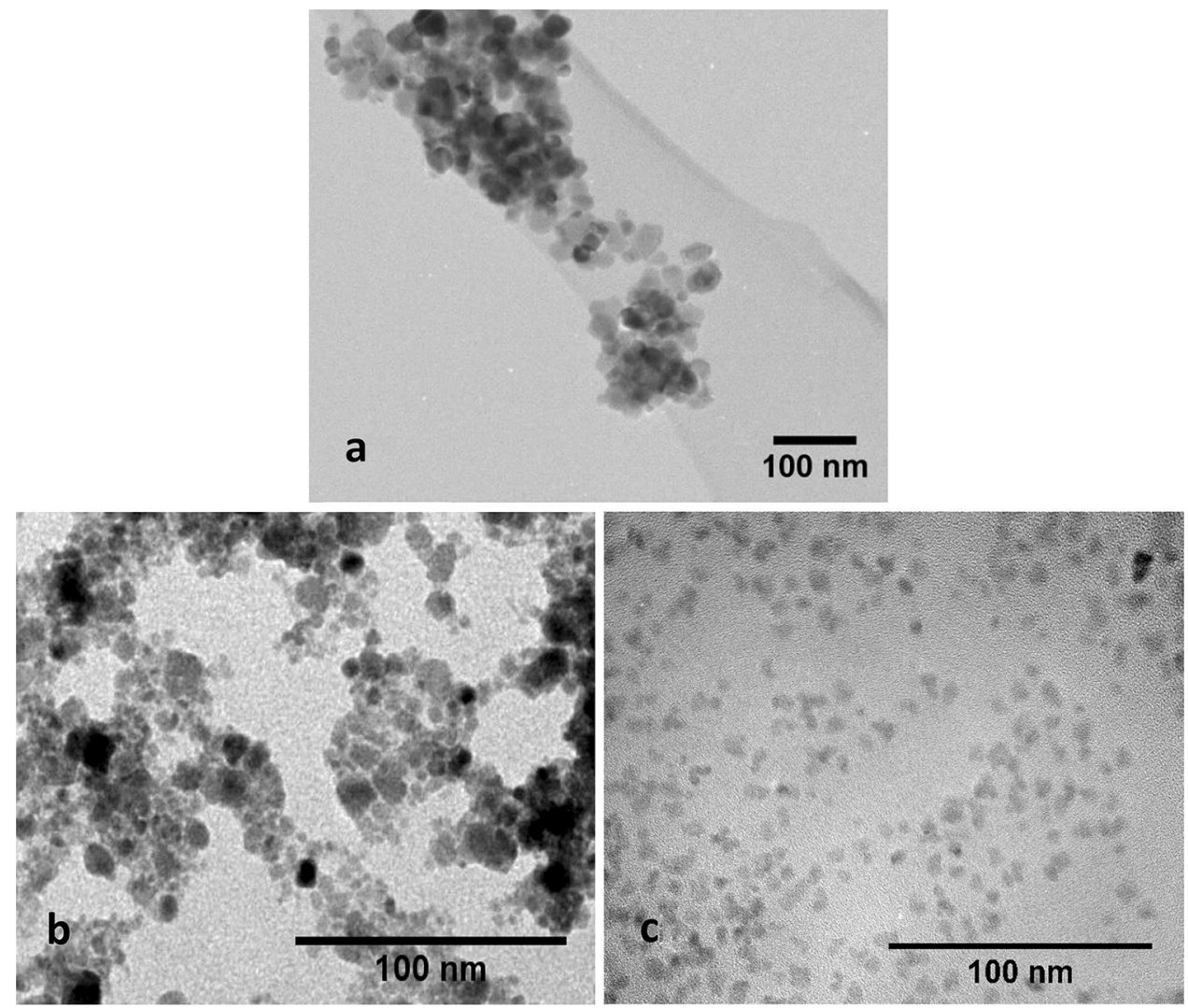

nanoparticles, they are greatly attracted to each other due to the magnetic dipole moment and disaggregation is almost impossible as there is no surface coating or surfactant.

The sample prepared by co-precipitation followed by an acid treatment, $\mathrm{CoFe}_{2} \mathrm{O}_{4}$ acid, presents a moderate dispersion and aggregation (Fig. 1b). The nanoparticles sizes range from 5 to $18 \mathrm{~nm}(\sigma=0.61)$. As can be noticed, the nanoparticles from this sample are smaller in size than the particles formed in the previous sample $\left(\mathrm{CoFe}_{2} \mathrm{O}_{4}\right.$ TT), because this time there was no heat treatment favoring the formation of larger particles; in addition, the acid treatment can dissolve nanoparticles with smaller sizes, favoring a lower dispersion. Also, this treatment gives a superficial charge to the nanoparticles [6]; this superficial charge reduces the agglomeration through Coulombic repulsive forces, improving the stability of the particles in water forming a water stable ferrofluid.

Finally, the third sample synthesized by a thermal decomposition procedure, $\mathrm{CoFe}_{2} \mathrm{O}_{4} \mathrm{TD}$, produced nanoparticles with the lowest size aggregation and dispersion (Fig. 1c). These results can be attributed to the use of surfactants (oleic acid and CTAB), controlling the size of the formed nanoparticles and avoiding their aggregation. The organic surfactants that cover the nanoparticles make them stable in nonpolar solvents, such as isooctane or toluene, forming a stable non-aqueous ferrofluid. While the size of the formed nanoparticles in this sample vary in between 4 and $14 \mathrm{~nm}$, most of them remain between 7 and $10 \mathrm{~nm}(\sigma=0.16)$. The histograms from all the samples are shown in Fig. 2.

Table 1 is derived from the EDS measurements showing the relationship between $\mathrm{Co} / \mathrm{Fe}$, as the stoichiometry is $1: 2$, the theoretical atomic ratio should be 0.5 . The sample TT has the closest values with respect to the theoretical ones (14.28\% Co and $28.57 \% \mathrm{Fe}$ ). However, the acid sample is deviated from the theoretical value, due to an excess of iron. This excess of iron is introduced at the acid treatment stage. Finally, the TD sample is also slightly deviated from the theoretical values due to the presence of the organic matter.

\subsection{Hydrodynamic volume}

As the samples synthesized by co-precipitation followed by an acid treatment, $\mathrm{CoFe}_{2} \mathrm{O}_{4}$ acid, and thermal decomposition, $\mathrm{CoFe}_{2} \mathrm{O}_{4} \mathrm{TD}$, are dispersible in polar and nonpolar solvents, respectively, a DLS analysis showed the stability and aggregation in liquid phases. The hydrodynamic volume $\left(V_{h}\right)$ determined from the DLS measurements show higher values than TEM measurements in accordance with previous reports; as the DLS size rarely 

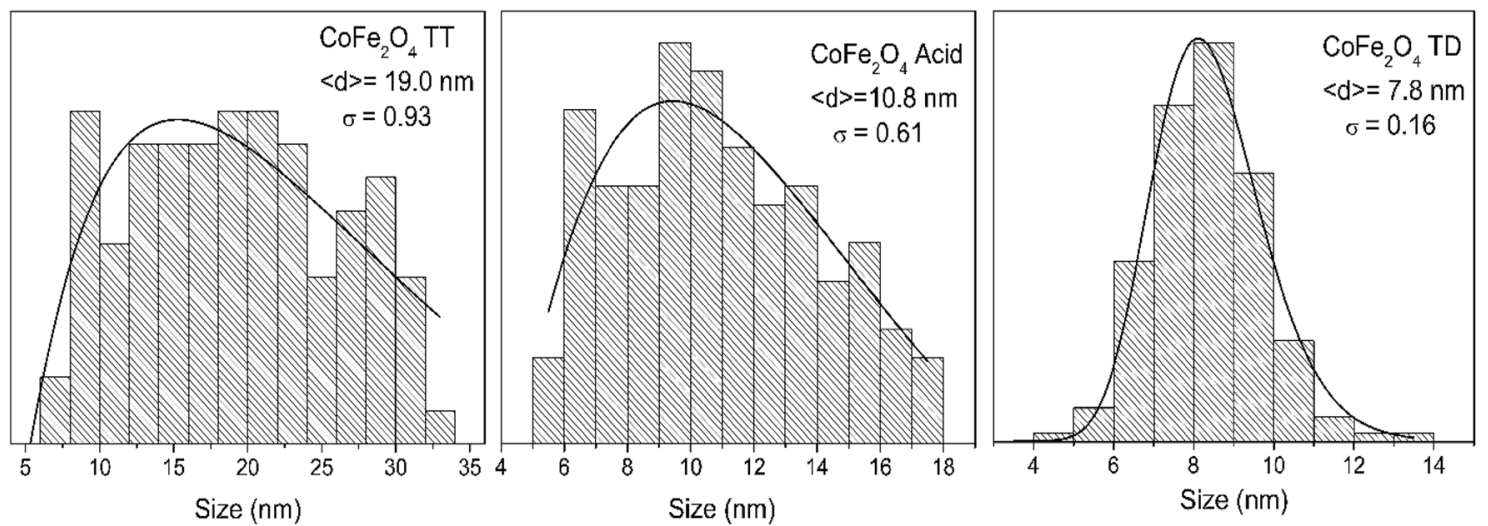

Fig. 2 Size distribution histograms of the nanoparticles fabricated using three synthetic routes. The average diameter $(<\mathrm{d}>)$ and standard deviation $(\sigma)$ were obtained by a fitting to a lognormal distribution

Table 1 Atomic \% of iron and cobalt in the samples

\begin{tabular}{llll}
\hline & Cobalt atomic \% & Iron atomic \% & $\begin{array}{l}\text { Relation- } \\
\text { ship Co/ } \\
\text { Fe }\end{array}$ \\
\hline $\mathrm{CoFe}_{2} \mathrm{O}_{4} \mathrm{TT}$ & 13.12 & 26.27 & 0.499 \\
$\mathrm{CoFe}_{2} \mathrm{O}_{4}$ acid & 10.32 & 32.38 & 0.318 \\
$\mathrm{CoFe}_{2} \mathrm{O}_{4} \mathrm{TD}$ & 11.9 & 30.86 & 0.385 \\
\hline
\end{tabular}

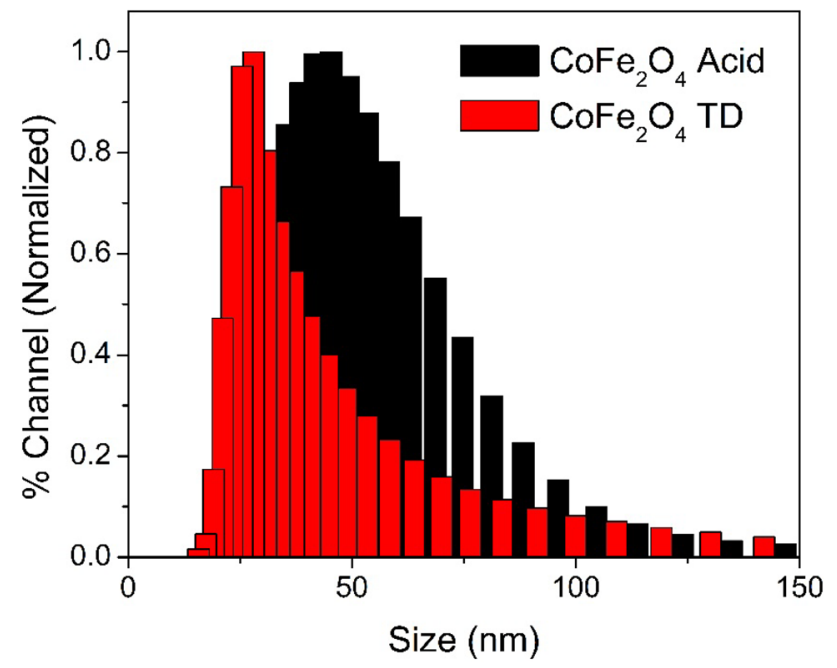

Fig. 3 DLS measurements of the samples $\mathrm{CoFe}_{2} \mathrm{O}_{4}$ acid and $\mathrm{CoFe}_{2} \mathrm{O}_{4} \mathrm{TD}$

correlates with the TEM measurements, especially for magnetic nanoparticles [25]. The DLS graph show that although there is some small degree of aggregation in the liquid phase, the hydrodynamic sizes of the particles are still in the nanometer range and form a stable ferrofluid in their respective media, as shown in Fig. 3. In addition, it can be clearly seen that sample $\mathrm{CoFe}_{2} \mathrm{O}_{4}$
TD has narrower and smaller sizes than sample $\mathrm{CoFe}_{2} \mathrm{O}_{4}$ acid; this behavior is in complete agreement with what is observed in TEM (Fig. 1).

\subsection{Crystalline structure}

All the synthesis mentioned above produced pure cobalt ferrite as indicated by the X-ray diffraction patterns shown in Fig. 4. All the revealed diffraction peaks shown in the diffractograms correspond to the spinel structure (PDF card \# 22-1086), and there are no other peaks indicating the formation of a different structure. It can be noticed that the most defined peaks are for $\mathrm{CoFe}_{2} \mathrm{O}_{4} \mathrm{TT}$, which is the sample with larger particle sizes.

In addition, it can be seen that samples $\mathrm{CoFe}_{2} \mathrm{O}_{4}$ TT and $\mathrm{CoFe}_{2} \mathrm{O}_{4}$ acid present a positive slope of their background due to the X-ray fluorescence of the sample when $\mathrm{Cu}$ radiation interacts with $\mathrm{Co}$ and $\mathrm{Fe}$. On the other hand, sample $\mathrm{CoFe}_{2} \mathrm{O}_{4}$ TD shows a negative slope in its background due to the organic compounds covering the nanoparticles. From Eq. 1, the average crystallite sizes calculated resulted in the following values: $16.6 \mathrm{~nm}$ for $\mathrm{CoFe}_{2} \mathrm{O}_{4}$ TT, $14.9 \mathrm{~nm}$ for $\mathrm{CoFe}_{2} \mathrm{O}_{4}$ acid and $8.5 \mathrm{~nm}$ for $\mathrm{CoFe}_{2} \mathrm{O}_{4}$ TD. This tendency is in agreement with those observed in the TEM micrographs.

\subsection{TGA}

The total organic mass for the sample $\mathrm{CoFe}_{2} \mathrm{O}_{4}$ TD was estimated to be $40 \%$ of the total mass, and this can be seen at the TGA of this sample at Fig. 5.

Figure 6 shows the results of DSC and TGA analysis with an applied external magnetic field for the three samples: $\mathrm{CoFe}_{2} \mathrm{O}_{4}$ TT, $\mathrm{CoFe} \mathrm{O}_{4}$ acid, and $\mathrm{CoFe}_{2} \mathrm{O}_{4}$ TD. The thermograms of Fig. $6 \mathrm{a}-\mathrm{c}$ show that the Curie temperature is almost independent of the size dispersion and 


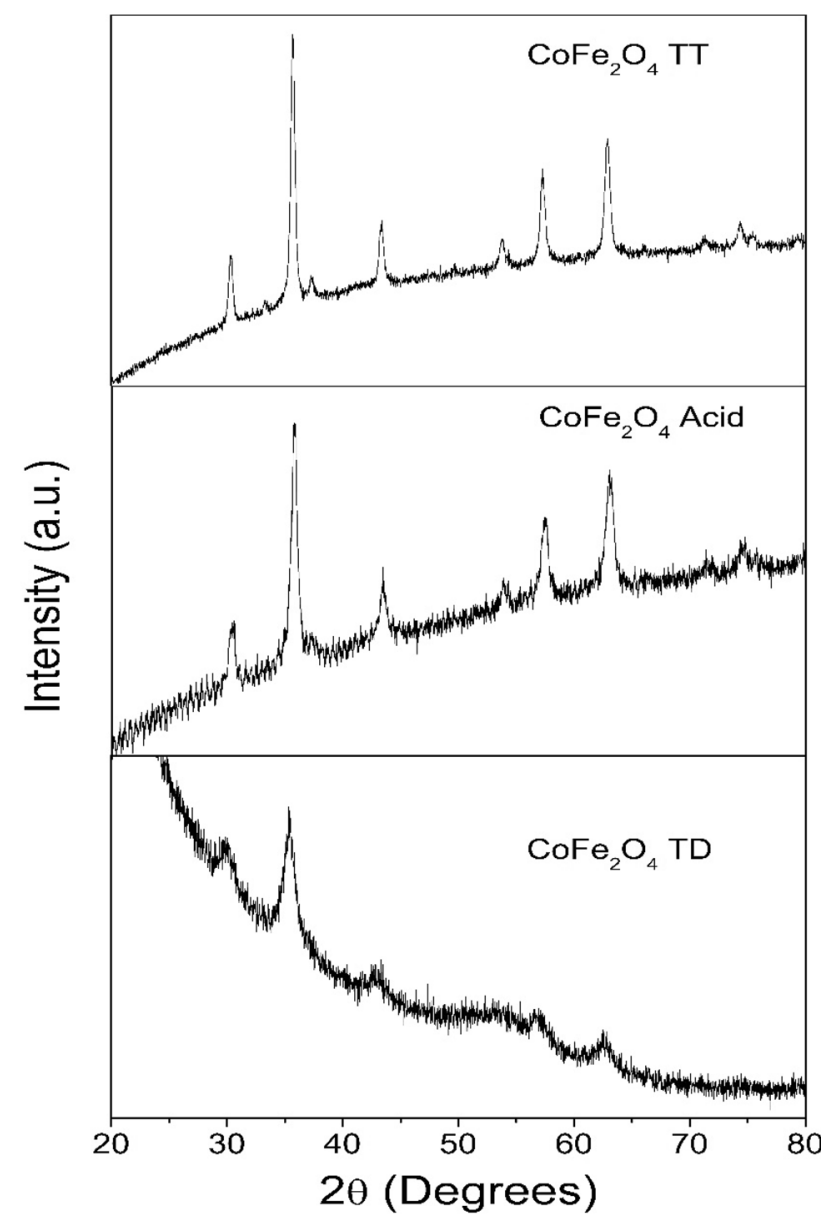

Fig. 4 X-ray diffraction patterns of samples $\mathrm{CoFe}_{2} \mathrm{O}_{4} \mathrm{TT}$, $\mathrm{CoFe}_{2} \mathrm{O}_{4}$ acid, and $\mathrm{CoFe}_{2} \mathrm{O}_{4}$ TD

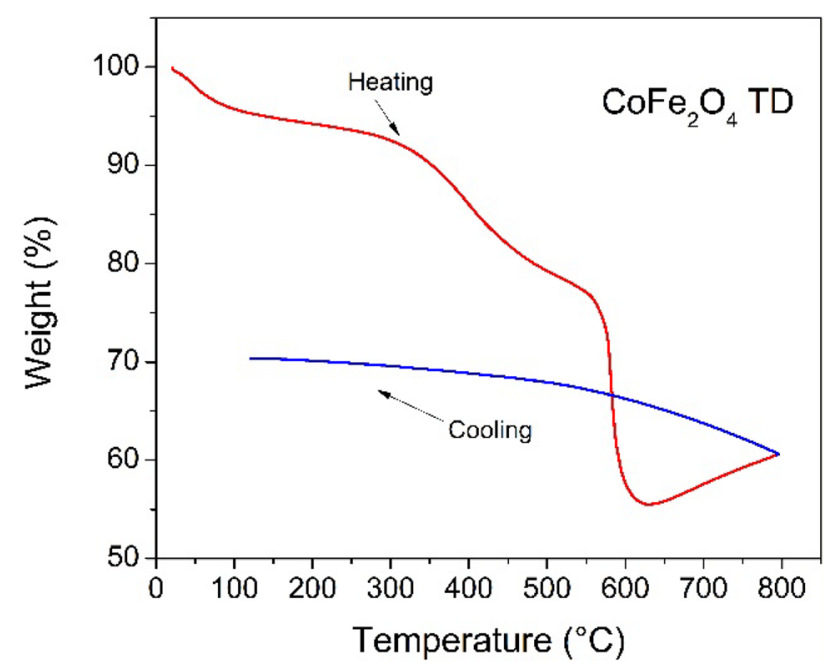

Fig. 5 TGA of sample $\mathrm{CoFe}_{2} \mathrm{O}_{4}$ TD used to determine the total organic mass aggregation, considering that the experimental ferromagnetic Curie temperature of the three samples keeps unchanged around $500{ }^{\circ} \mathrm{C}$, even for the second scan where the water and organic components were already removed. These results suggest that the Curie temperature is a property more related to the crystalline structure and the composition than to the size of the nanoparticles.

\subsection{Magnetometry}

The magnetization hysteresis curves (Fig. 7) show the characteristic high coercivity of the cobalt ion $[15,31]$. In this case, the magnetic properties showed a strong relationship with the size and dispersion of the nanoparticles. The hysteresis curve of sample $\mathrm{CoFe}_{2} \mathrm{O}_{4}$ TT at $10 \mathrm{~K}$ showed a well-defined step (indicated by arrows in Fig. 7). The step decreased for sample $\mathrm{CoFe}_{2} \mathrm{O}_{4}$ acid, while for sample $\mathrm{CoFe}_{2} \mathrm{O}_{4}$ TD it completely disappeared. It can be hypothesized that this step at low temperatures in the hysteresis curve is due to the size dispersion of the nanoparticles. This behavior can be explained because at low temperatures, the magnetic moments are completely frozen when they aligned to the magnetic field and because smaller nanoparticles have lower saturation values, they reach a lower saturation; so the step shown at low temperatures is possible because there is a group of smaller nanoparticles that reached the saturation point, while the largest nanoparticles have not reached saturation yet.

The step diminishes as the temperature rises, because there is no full alignment of the nanoparticle spins, as the thermal energy becomes more significative and it is enough to partially dis-align the magnetic moment. These jumps in the hysteresis curves have been previously observed in other articles [4, 34, 37], although there was not much discussion about its origin.

The highest saturation magnetization measured at $300 \mathrm{~K}$ was for sample $\mathrm{CoFe}_{2} \mathrm{O}_{4}$ TT at $62 \mathrm{emu} / \mathrm{g}$, while the lowest was for sample $\mathrm{CoFe}_{2} \mathrm{O}_{4}$ TD at $21 \mathrm{emu} / \mathrm{g}$. Sample $\mathrm{CoFe}_{2} \mathrm{O}_{4}$ acid had an intermediate saturation value at $56 \mathrm{emu} / \mathrm{g}$. Sample $\mathrm{CoFe}_{2} \mathrm{O}_{4}$ TT had the highest saturation value, because of two important factors: It has the best crystallinity as revealed from its XRD pattern (Fig. 4, appearing most intense and well-defined diffraction peaks), due to the post-synthesis thermal treatment; the second factor is that this sample had the largest nanoparticles size. In addition, sample $\mathrm{CoFe}_{2} \mathrm{O}_{4}$ TD had the lowest saturation value because of the non-magnetic mass contribution from the organic surfactants (oleic acid and CTAB).

Figure 8 shows a magnification of the coercivity values of the samples; those values decreased with respect to the temperature as expected, but the values are completely different from one sample to another. The superparamagnetic behavior of sample $\mathrm{CoFe}_{2} \mathrm{O}_{4}$ TD is seen also in Fig. 8, for the 

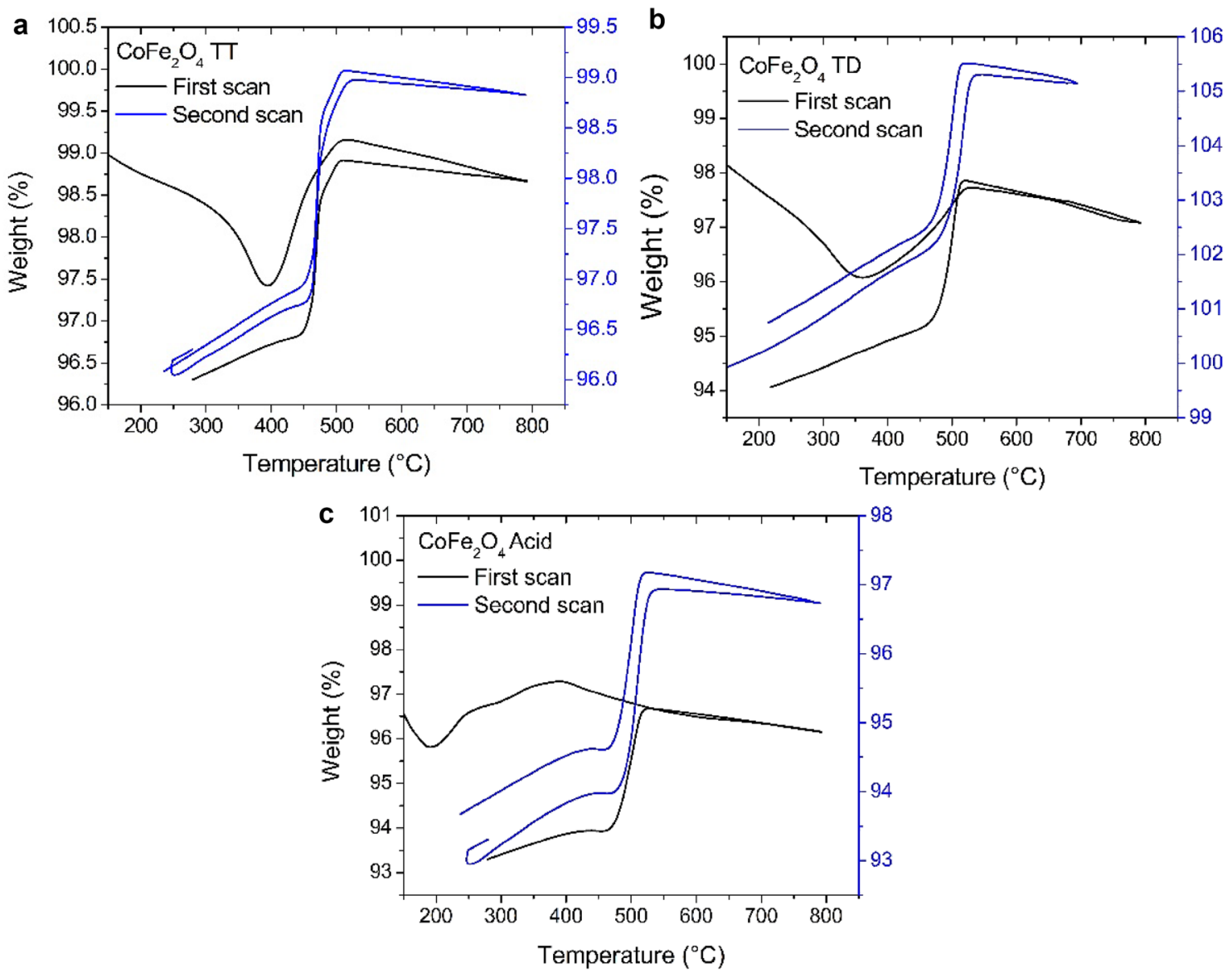

Fig. 6 TGA of samples $\mathrm{CoFe}_{2} \mathrm{O}_{4} \mathrm{TT}(\mathbf{a}), \mathrm{CoFe}_{2} \mathrm{O}_{4} \mathrm{TD}(\mathbf{b}), \mathrm{CoFe}_{2} \mathrm{O}_{4}$ acid (c) showing the Curie temperature transition on heating and cooling around $500{ }^{\circ} \mathrm{C}$

reason that the curve at $300 \mathrm{~K}$ and at $350 \mathrm{~K}$ (blue and black, respectively) completely overlaps, at the shown area.

\subsection{Blocking temperature determination}

The blocking temperature cannot be determined from the ZFC-FC curves of samples $\mathrm{CoFe}_{2} \mathrm{O}_{4}$ TT and $\mathrm{CoFe}_{2} \mathrm{O}_{4}$ acid. $\mathrm{CoFe}_{2} \mathrm{O}_{4}$ TT shows an inversion of the ZFC-FC curves due to a large magnetostriction effect in the whole range of the measurement (discussed elsewhere [37]), and $\mathrm{CoFe}_{2} \mathrm{O}_{4}$ acid revealed no clear maximum in the ZFC curve, meaning that the blocking temperature is over $350 \mathrm{~K}$. On the other hand, sample $\mathrm{CoFe}_{2} \mathrm{O}_{4}$ TD reveals a clear blocking temperature value of $248 \mathrm{~K}$ under at 100 Oe applied magnetic field [38].

The critical volume $\left(V_{c}\right)$ to achieve a superparamagnetic state for cobalt ferrite can be calculated by using the blocking temperature $\left(T_{\mathrm{B}}\right)$ Eq. (2) [5]:

$T_{\mathrm{B}}=\frac{V_{\mathrm{c}} K_{\text {eff }}}{k_{\mathrm{B}} \ln \left(\tau_{\mathrm{m} / \tau_{0}}\right)}$ where $V_{c}$ is the critical volume of the nanoparticles, $k_{B}$ is the Boltzmann constant, $\tau_{\mathrm{m}}$ is the inverse of the measuring frequency and $\tau_{0}$ the attempt time, usually the natural logarithm value is approximated to $\approx 25$ and $K_{\text {eff }}$ is the effective anisotropy of cobalt ferrite, by using the reported bulk cobalt ferrite anisotropy and calculated values for the cobalt ferrite samples in Eq. (3) [12]:

$H_{\mathrm{c}}=\left(\frac{2 K_{\mathrm{eff}}}{\mu_{0} M_{\mathrm{s}}}\right)\left(1-\frac{25 k_{\mathrm{B}} T}{K_{\mathrm{eff}} V}\right)$

where $H_{c}$ is the coercivity field, $M_{s}$ is the saturation magnetization and $T$ the temperature. It can be found that the $V_{c}$ to ensure a superparamagnetic state for the cobalt ferrite is in the range of 9 to $12 \mathrm{~nm}$ depending on the effective anisotropy of the material, as it usually increases as the nanoparticles size increases. However, as shown in Figs. 8 and 9 , there is no evidence of superparamagnetism in the samples $\mathrm{CoFe}_{2} \mathrm{O}_{4}$ TT and $\mathrm{CoFe}_{2} \mathrm{O}_{4}$ acid. In Fig. 8, it can be seen that there is still considerable coercivity at $300 \mathrm{~K}$ and above, except for sample $\mathrm{CoFe}_{2} \mathrm{O}_{4}$ TD. 

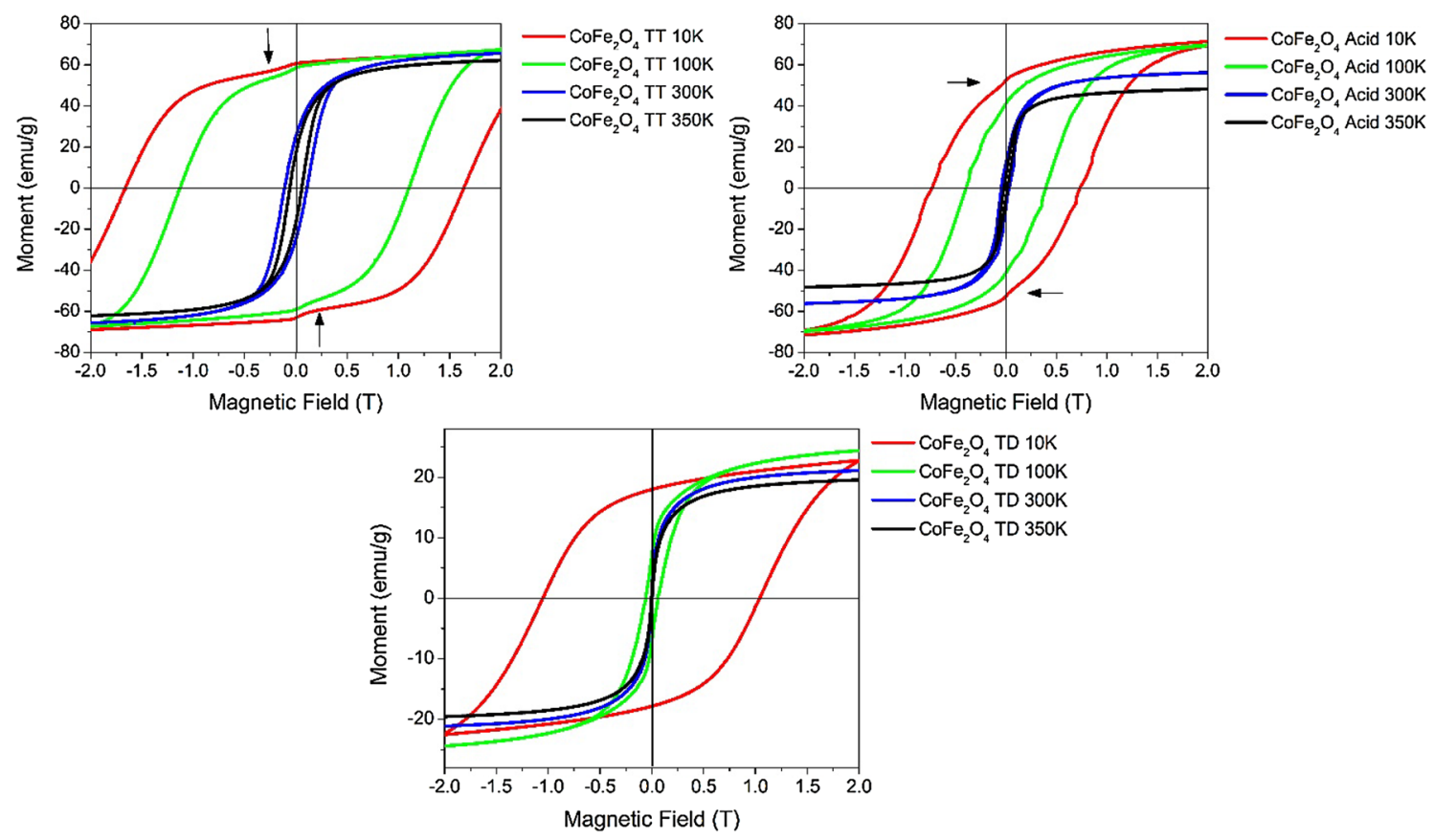

- $\mathrm{CoFe}_{2} \mathrm{O}_{4}$ TD $10 \mathrm{~K}$

$-\mathrm{CoFe}_{2} \mathrm{O}_{4} \mathrm{TD} 100 \mathrm{~K}$ $-\mathrm{CoFe}_{2} \mathrm{O}_{4}$ TD $350 \mathrm{~K}$

Fig. 7 Hysteresis curves for the different samples at three temperatures
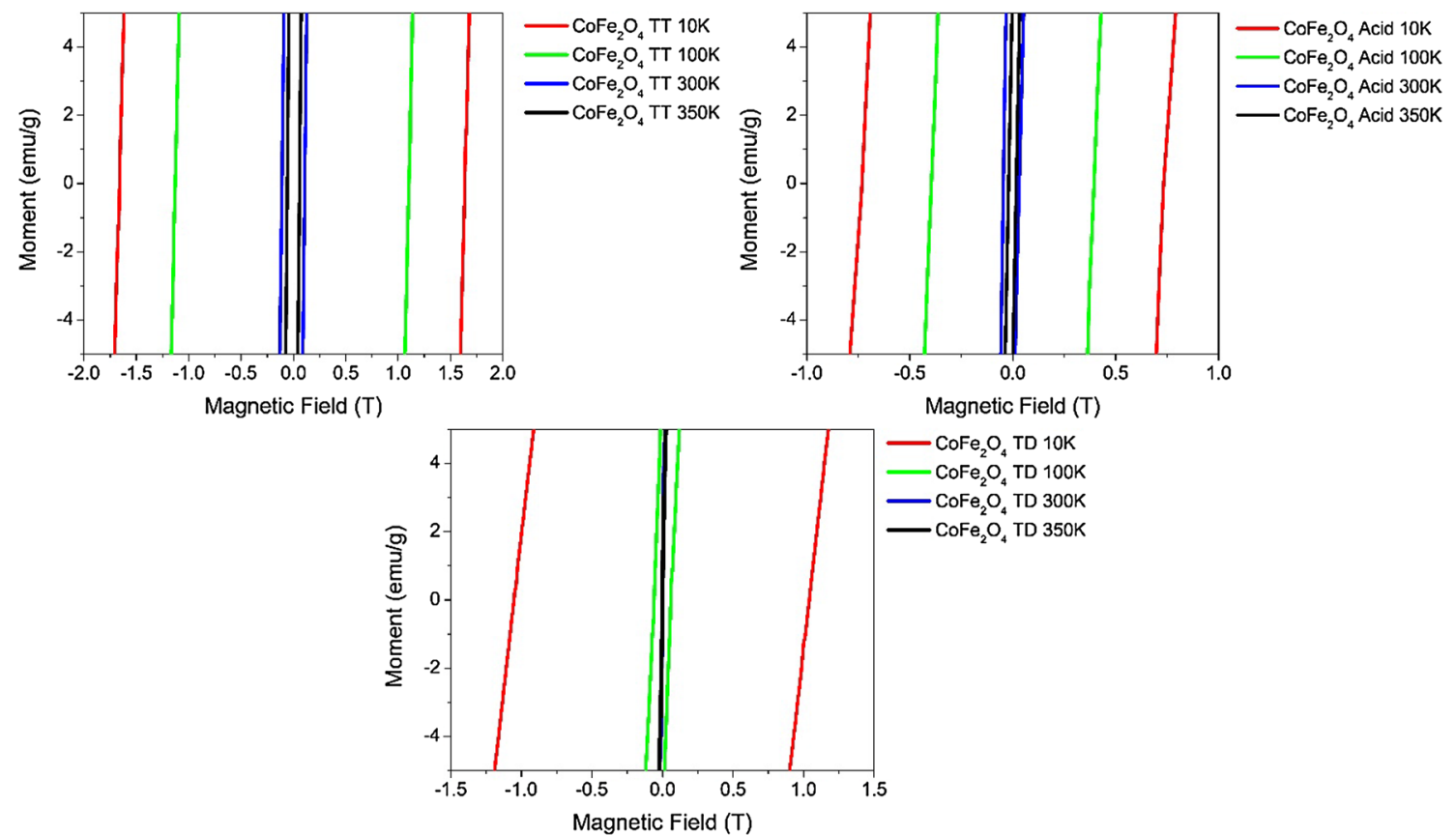

- $\mathrm{COFe}_{2} \mathrm{O}_{4} \mathrm{TD} 10 \mathrm{~K}$ $-\mathrm{CoFe}_{2} \mathrm{O}_{4}$ TD $100 \mathrm{~K}$ $-\mathrm{CoFe}_{2} \mathrm{O}_{4}$ TD $300 \mathrm{~K}$ $-\mathrm{COFe}_{2} \mathrm{O}_{4}$ TD $350 \mathrm{~K}$

Fig. 8 Amplification of the hysteresis curves for all samples, showing their coercivity values of the fabricated nanoparticles at different temperatures

As TEM images indicated (Fig. 1), nanoparticles small enough to be in the superparamagnetic state are present in all the samples, but for $\mathrm{CoFe}_{2} \mathrm{O}_{4} \mathrm{TT}$ and $\mathrm{CoFe}_{2} \mathrm{O}_{4}$ acid the ZFC curves (Fig. 9) showed no significant contribution from these small nanoparticles, indicating that the properties of the non-superparamagnetic 

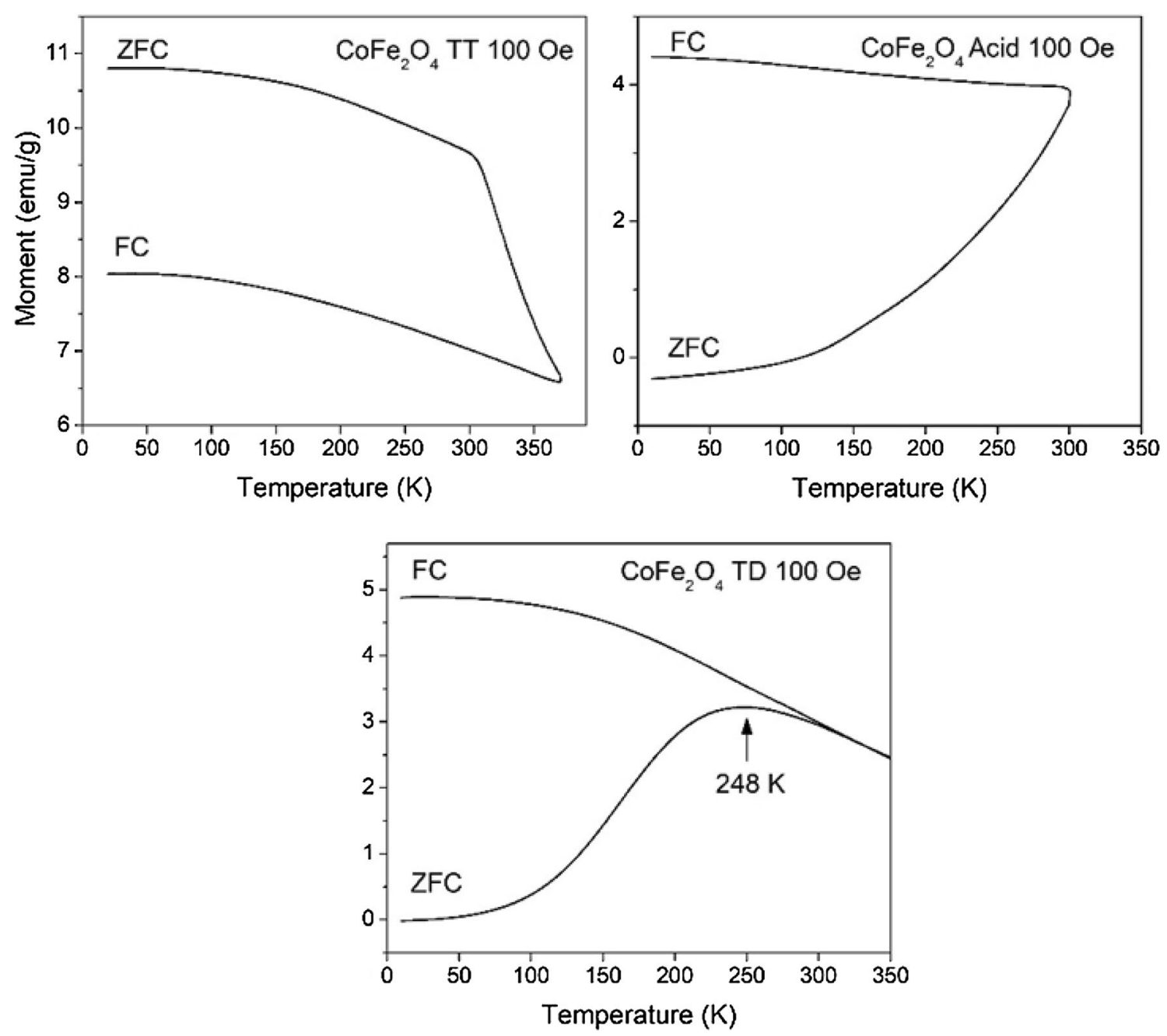

Fig. 9 ZFC-FC curves of the samples

larger nanoparticles dominate the measurements, hiding the contribution of the smaller nanoparticles; it also suggests that agglomeration may be preventing their superparamagnetic contribution. This effect is achieved by increasing the effective anisotropy values through an interparticle interaction energy [7, 32].

As sample $\mathrm{CoFe}_{2} \mathrm{O}_{4}$ TD does not present a significant population of larger nanoparticles and the nanoparticles are well dispersed, the interparticle interaction energy should be very low [11]; in this case, the major contribution arises from the nanoparticles around $8 \mathrm{~nm}$. For that reason, the ZFC curves for this sample indeed present a clear superparamagnetic state as expected from the calculated values for the critical volume for superparamagnetism. Table 2 summarizes the magnetic properties for all the samples.

Table 2 Values of the magnetic properties for all the samples

\begin{tabular}{|c|c|c|c|c|c|c|c|c|c|c|c|}
\hline & \multirow[t]{2}{*}{ Dispersion $(\sigma)$} & \multicolumn{4}{|c|}{ Magnetic saturation (emu/g) } & \multicolumn{4}{|c|}{ Coercivity (T) } & \multirow{2}{*}{$\begin{array}{l}\text { Curie tem- } \\
\text { perature }\left({ }^{\circ} \mathrm{C}\right)\end{array}$} & \multirow{2}{*}{$\begin{array}{l}\text { Blocking } \\
\text { temperature } \\
\text { (K) }\end{array}$} \\
\hline & & $10 \mathrm{~K}$ & $100 \mathrm{~K}$ & $300 \mathrm{~K}$ & $350 \mathrm{~K}$ & $10 \mathrm{~K}$ & $100 \mathrm{~K}$ & $300 \mathrm{~K}$ & $350 \mathrm{~K}$ & & \\
\hline $\mathrm{CoFe}_{2} \mathrm{O}_{4} \mathrm{TT}$ & 0.93 & - & 68 & 66 & 61 & 1.6 & 1.1 & 0.11 & 0.5 & 460 & - \\
\hline $\mathrm{CoFe}_{2} \mathrm{O}_{4}$ Acid & 0.61 & 71 & 70 & 57 & 48 & 1.0 & 0.39 & 0.047 & 0.016 & 465 & - \\
\hline $\mathrm{CoFe}_{2} \mathrm{O}_{4} \mathrm{TD}$ & 0.16 & 22 & 24 & 21 & 19 & 0.72 & 0.058 & 0.003 & 0.0017 & 467 & 248 \\
\hline
\end{tabular}



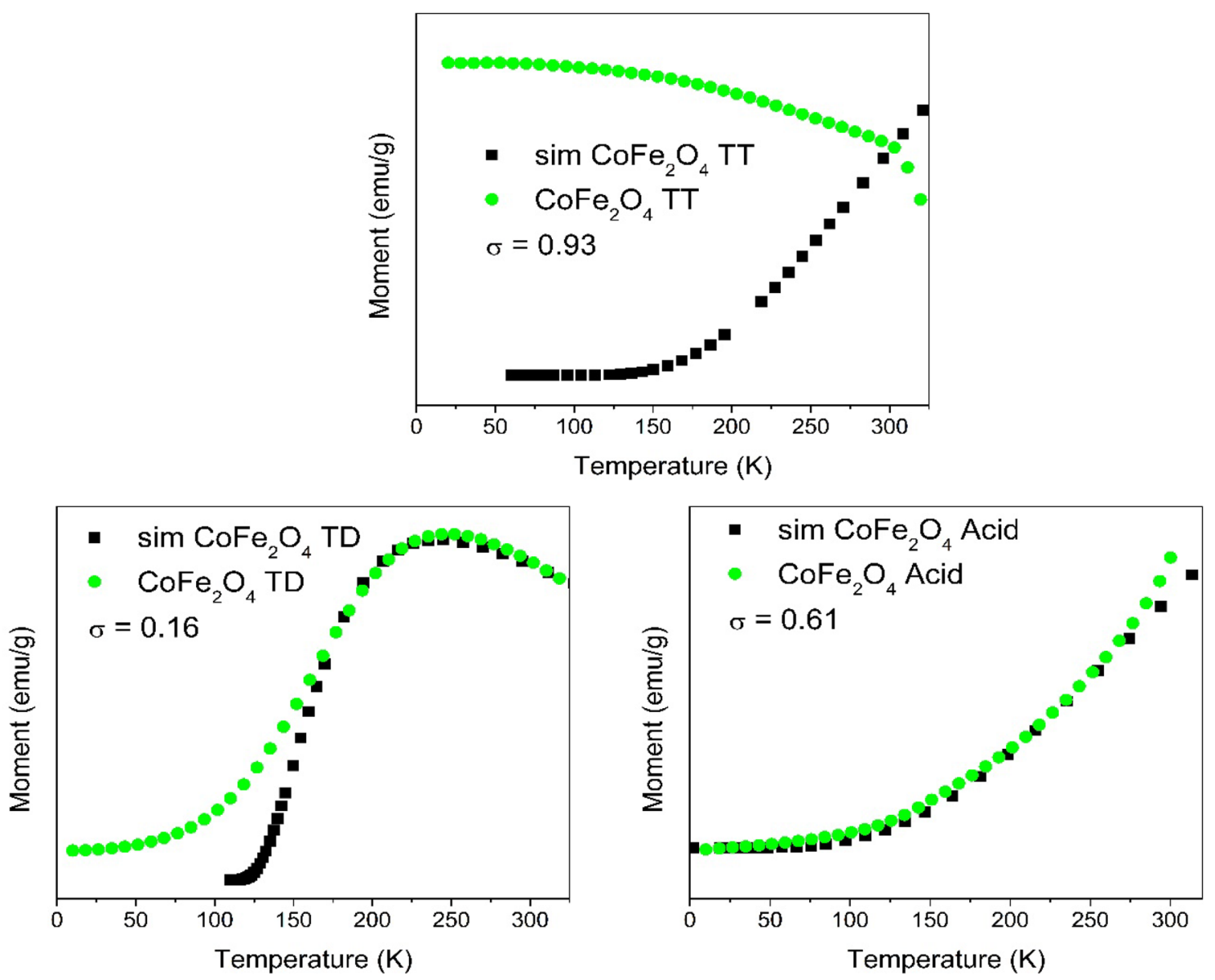

Fig. 10 Experimental (green curves in circles) and simulated (black curves in squares) ZFC curves for all samples

\subsection{Stoner-Wohlfarth model for ZFC simulation}

Simulation curves of the ZFC of the samples were performed in order to compare our experimental results with what the theory predicts on how the size dispersion $(\sigma)$ affects the ZFC, and thus the blocking temperature. For the simulation, a Stoner-Wohlfarth (SW) model was used considering thermal fluctuations; this model is reported elsewhere [8]. The simulation was performed by solving Eq. 4, for a linear temperature variation with slope $B\left(T(t)=B t+t_{0}\right)$, and introducing the size dispersion of our samples, through a linear addition of magnetization values from the given sizes, obtained by fitting the histogram to a lognormal distribution.

$\frac{\mathrm{d} m}{\mathrm{~d} T}=\frac{2}{B \tau_{0}} e^{-E\left(1+h^{2}\right)}[\sinh (2 E h)-m \cosh (2 E h)]$

$E=\frac{E_{\mathrm{B}}}{E_{\mathrm{T}}}$
$h=\frac{H}{H_{\mathrm{k}}}=\frac{H \mu_{0} M_{\mathrm{s}}}{2 K_{\text {eff }}}$

The experimental and simulated ZFC curves for all the samples are shown in Fig. 10. The variation of the $\sigma$ parameter in the simulation according to those obtained from the lognormal distribution correlates with the experimentally obtained curves. Due to small changes in $\sigma$, and to the high anisotropy induced by cobalt atoms within the structure, the blocking temperature moved to high temperatures, above room temperature; this result is well known as there are few articles reporting an experimental blocking temperature for cobalt ferrite, as a consequence of its high blocking temperature; that behavior is clearly seen in the simulation of the ZFC curves, where the simulation of the sample $\mathrm{CoFe}_{2} \mathrm{O}_{4}$ acid does not show any signs of a blocking temperature near room temperature. Since the used model does not take into account any magnetostriction parameters, the fitting of the simulation with the experimental was unsuccessful for sample $\mathrm{CoFe}_{2} \mathrm{O}_{4}$ TT. 


\section{Conclusion}

In conclusion, size dispersion and aggregation are always present in the samples synthesized by chemical procedures in different degrees, depending on the chosen synthesis route, affecting in a direct way its magnetic properties. As we demonstrate, there are some properties that are not affected by the dispersion and particle aggregation like the Curie temperature. Nevertheless, the hysteresis loops, ZFC-FC curves, and the saturation magnetization and blocking temperature of the magnetic materials are strongly affected by size dispersion and particle aggregation.

Acknowledgements This work was supported by CONACyT, Mexico, through the Grant \# INFR-2014-02-23053.

\section{Compliance with ethical standards}

Conflict of interest The authors declare no competing financial interests.

\section{References}

1. Afroz M, Karthikeyan P, Ahmed P, Kumar U (2012) Application of nanotechnology in food and dairy processing: an overview. Pak J Food Sci 22:23-31

2. Ahn T, Kim JH, Yang HM et al (2012) Formation pathways of magnetite nanoparticles by coprecipitation method. J Phys Chem C 116:6069-6076. https://doi.org/10.1021/jp211843g

3. Arteaga-Cardona F, Hidalgo-Tobón S, Pal U, Méndez-Rojas MÁ (2016) Ferrites as magnetic fluids for hyperthermia and MRI contrast agents. In: AIP conference proceedings

4. Arteaga-Cardona F, Rojas-Rojas K, Costo R et al (2016) Improving the magnetic heating by disaggregating nanoparticles. J Alloys Compd. https://doi.org/10.1016/j.jallcom.2015.10.285

5. Arteaga-Cardona F, Santillán-Urquiza E, Pal U et al (2017) Unusual variation of blocking temperature in bi-magnetic nanoparticles. J Magn Magn Mater 441:417-423. https://doi. org/10.1016/j.jmmm.2017.06.024

6. Auzans E, Zins D, Blums E, Massart R (1999) Synthesis and properties of Mn-Zn ferrite ferrofluids. J Mater Sci 34:1253-1260. https://doi.org/10.1023/A:1004525410324

7. Branquinho LC, Carrião MS, Costa AS et al (2013) Effect of magnetic dipolar interactions on nanoparticle heating efficiency: implications for cancer hyperthermia. Sci Rep 3:2887. https:// doi.org/10.1038/srep02887

8. Bruvera IJ, Calatayud MP, Goya GF (2015) Determination of the blocking temperature of magnetic nanoparticles: the good, the bad and the ugly. J Appl Phys 118:1-8. https://doi. org/10.1063/1.4935484

9. Cardona FA, Urquiza ES, de la Presa P et al (2016) Enhanced magnetic properties and MRI performance of bi-magnetic core-shell nanoparticles. RSC Adv 6:77558-77568. https://doi.org/10.1039/ C6RA14265F

10. Chandekar KV, Kant KM (2017) Effect of size and shape dependent anisotropy on superparamagnetic property of $\mathrm{CoFe}_{2} \mathrm{O}_{4}$ nanoparticles and nanoplatelets. Phys B Phys Condens Matter 520:152-163. https://doi.org/10.1016/j.physb.2017.06.001

11. Crespo $P$ (2013) Tuning properties with coatings. J Phys Condens Matter. https://doi.org/10.1088/0953-8984/25/48/484006

12. De La Presa P, Luengo Y, Multigner M et al (2012) Study of heating efficiency as a function of concentration, size, and applied field in $\mathrm{\gamma}-\mathrm{Fe}_{2} \mathrm{O}_{3}$ nanoparticles. J Phys Chem C 116:25602-25610

13. Dorozhkin SV (2010) Nanosized and nanocrystalline calcium orthophosphates. Acta Biomater 6:715-734. https://doi. org/10.1016/j.actbio.2009.10.031

14. Dutz S, Hergt R (2014) Magnetic particle hyperthermia-a promising tumour therapy? Nanotechnology 25:452001. https ://doi.org/10.1088/0957-4484/25/45/452001

15. Fantechi E, Innocenti C, Albino M et al (2015) Influence of cobalt doping on the hyperthermic efficiency of magnetite nanoparticles. J Magn Magn Mater 380:365-371. https://doi. org/10.1016/j.jmmm.2014.10.082

16. Gutiérrez L, Costo R, Grüttner C et al (2015) Synthesis methods to prepare single- and multi-core iron oxide nanoparticles for biomedical applications. Dalt Trans 44:2943-2952. https://doi. org/10.1039/C4DT03013C

17. Gyergyek S, Drofenik M, Makovec D (2012) Oleic-acid-coated $\mathrm{CoFe}_{2} \mathrm{O}_{4}$ nanoparticles synthesized by co-precipitation and hydrothermal synthesis. Mater Chem Phys 133:515-522. https ://doi.org/10.1016/j.matchemphys.2012.01.077

18. Hasier J, Riolo MA, Nash P (2017) Curie temperature determination via thermogravimetric and continuous wavelet transformation analysis. EPJ Tech Instrum 4:5. https://doi. org/10.1140/epjti/s40485-017-0040-y

19. Khalil MI (2015) Co-precipitation in aqueous solution synthesis of magnetite nanoparticles using iron (III) salts as precursors. Arab J Chem 8:279-284. https://doi.org/10.1016/j.arabj c.2015.02.008

20. Kiss LB, Niklasson GA, Granqvist CG (1999) New approach to the origin of lognormal size distributions of nanoparticles. Nanotechnology 10:25-28

21. Langford Jl, Wilson AJC (1978) Scherrer after sixty years: a survey and some new results in the determination of crystallite size. J Appl Crystallogr 11:102-113. https://doi.org/10.1107/ S0021889878012844

22. Laokul P, Arthan S, Maensiri S, Swatsitang E (2015) Magnetic and optical properties of $\mathrm{CoFe}_{2} \mathrm{O}_{4}$ nanoparticles synthesized by reverse micelle microemulsion method. J Supercond Nov Magn 28:2483-2489. https://doi.org/10.1007/s1094 8-015-3068-8

23. Leite GCP, Chagas EF, Pereira R et al (2012) Exchange coupling behavior in bimagnetic $\mathrm{CoFe}_{2} \mathrm{O}_{4} / \mathrm{CoFe}_{2}$ nanocomposite. $\mathrm{J}$ Magn Magn Mater 324:2711-2716. https://doi.org/10.1016/J. Jmmm.2012.03.034

24. Lemine OM, Omri K, Iglesias $\mathrm{M}$ et al (2014) $\mathrm{Y}-\mathrm{Fe}_{2} \mathrm{O}_{3}$ by sol-gel with large nanoparticles size for magnetic hyperthermia application. J. Alloys Compd 607:125-131

25. Lim J, Yeap S, Che H, Low S (2013) Characterization of magnetic nanoparticle by dynamic light scattering. Nanoscale Res Lett 8:381. https://doi.org/10.1186/1556-276X-8-381

26. López-Ortega A, Estrader M, Salazar-Alvarez G et al (2015) Applications of exchange coupled bi-magnetic hard/soft and soft/ hard magnetic core/shell nanoparticles. Phys Rep 553:1-32. https://doi.org/10.1016/j.physrep.2014.09.007

27. Lu LT, Dung NT, Tung LD et al (2016) Synthesis of magnetic cobalt ferrite nanoparticles with controlled morphology, monodispersity and composition: the influence of solvent, surfactant, reductant and synthetic conditions. Nanoscale 7:19596-19610. https://doi.org/10.1039/c5nr04266f

28. Lu RE, Chang KG, Fu B et al (2014) Magnetic properties of different $\mathrm{CoFe}_{2} \mathrm{O}_{4}$ nanostructures: nanofibers versus nanoparticles. 
J Mater Chem C 2:8578-8584. https://doi.org/10.1039/c4tc0 $1415 d$

29. Mohapatra J, Mitra A, Bahadur D, Aslam M (2013) Surface controlled synthesis of MFe2O4 ( $\mathrm{M}=\mathrm{Mn}, \mathrm{Fe} \mathrm{Co}, \mathrm{Ni}$ and $\mathrm{Zn}$ ) nanoparticles and their magnetic characteristics. CrystEngComm 15:524-532. https://doi.org/10.1039/C2CE25957E

30. Monshi A, Foroughi MR, Monshi MR (2012) Modified Scherrer equation to estimate more accurately nano-crystallite size using XRD. World J Nano Sci Eng 02:154-160. https://doi.org/10.4236/ wjnse.2012.23020

31. Morais PC, Garg VK, Oliveira AC et al (2001) Synthesis and characterization of size-controlled cobalt-ferrite-based ionic ferrofluids. J Magn Magn Mater 225:37-40. https://doi.org/10.1016/ S0304-8853(00)01225-7

32. Ostenfeld C, Mørup S (2002) Magnetic interactions between nanoparticles of different materials. Hyperfine Interact. https ://doi.org/10.1007/978-94-010-0281-3_21

33. Papaefthymiou GC (2009) Nanoparticle magnetism. Nano Today 4:438-447. https://doi.org/10.1016/j.nantod.2009.08.006

34. Pereira C, Pereira AM, Fernandes C et al (2012) Superparamagnetic MFe2O4 ( $M=F e C o, M n)$ nanoparticles : tuning the particle size and magnetic properties through a novel one-step coprecipitation route. Chem Mater 24:1496-1504. https://doi. org/10.1021/cm300301c

35. Polte J (2015) Fundamental growth principles of colloidal metal nanoparticles-a new perspective. CrystEngComm 17:68096830. https://doi.org/10.1039/C5CE01014D

36. Salazar-kuri U, Estevez JO, Silva-gonzález NR et al (2017) Structure and magnetic properties of the $\mathrm{Co}_{1-\mathrm{x}} \mathrm{Ni}_{\mathrm{x}} \mathrm{Fe}_{2} \mathrm{O}_{4}-\mathrm{BaTiO}_{3}$ core-shell nanoparticles. J Magn Magn Mater 442:247-254. https://doi.org/10.1016/j.jmmm.2017.06.126

37. Salazar-kuri U, Estevez JO, Silva-gonzález NR, Pal U (2018) Large magnetostriction in chemically fabricated $\mathrm{CoFe}_{2} \mathrm{O}_{4}$ nanoparticles and its temperature dependence. J Magn Magn Mater 460:141-145. https://doi.org/10.1016/j.jmmm.2018.03.074

38. Sugiura N (1980) Field dependence of blocking temperature in magnetite nanoparticles. Earth Planet Sci Lett 46:438-442. https ://doi.org/10.4028/www.scientific.net/JMNM.20-21.673

39. Thanh NTK, Maclean N, Mahiddine S (2014) Mechanisms of nucleation and growth of nanoparticles in solution. Chem Rev 114:7610-7630. https://doi.org/10.1021/cr400544s

40. Tiwari A, Ramalingam M, Kobayashi H, Turner APF (2012) Biomedical materials and diagnostic devices. Wiley. https://doi. org/10.1002/9781118523025

41. Unni M, Uhl AM, Savliwala S et al (2017) Thermal decomposition synthesis of iron oxide nanoparticles with diminished magnetic dead layer by controlled addition of oxygen. ACS Nano 11:22842303. https://doi.org/10.1021/acsnano.7b00609

42. Wu W, Wu Z, Yu T et al (2015) Recent progress on magnetic iron oxide nanoparticles: synthesis, surface functional strategies and biomedical applications. Sci Technol Adv Mater 16:023501. https ://doi.org/10.1088/1468-6996/16/2/023501

43. Yigit MV, Moore A, Medarova Z (2012) Magnetic nanoparticles for cancer diagnosis and therapy. Pharm Res 29:1180-1188. https://doi.org/10.1007/s11095-012-0679-7

Publisher's Note Springer Nature remains neutral with regard to jurisdictional claims in published maps and institutional affiliations. 